\title{
Multiferroic behavior of heterostructures composed of lanthanum and bismuth ferrite
}

\author{
P.P. Ortega ${ }^{a}$, L.S.R. Rocha ${ }^{a}$, C.C. Silva ${ }^{a}$, M. Cilense ${ }^{b}$, R.A.C. Amoresi ${ }^{b}$, E. Longo ${ }^{b}$, \\ A.Z. Simões ${ }^{\text {a,* }}$ \\ ${ }^{a}$ Faculty of Engineering of Guaratinguetá, São Paulo State University - UNESP, Guaratinguetá, SP, 12516-410 Brazil \\ ${ }^{\mathrm{b}}$ Interdisciplinary Laboratory of Electrochemistry and Ceramics, LIEC - Chemistry Institute, São Paulo State University - UNESP, Araraquara - SP, Araraquara, \\ SP, 14800-060 Brazil
}

\section{A R T I C L E I N F O}

\section{Article history:}

Received 24 June 2016

Accepted 11 July 2016

Available online 12 July 2016

Keywords:

A. Films

C. Electrical properties

C. Magnetic properties

E. Functional applications

\begin{abstract}
A B S T R A C T
Heterostructured thin films of lanthanum ferrite (LFO) and bismuth ferrite (BFO) with different thicknesses were successfully obtained by a soft chemical method. The films were deposited by spin-coating and annealed at $500^{\circ} \mathrm{C}$ for $2 \mathrm{~h}$. The XRD pattern confirmed the purity of the thin films, where no additional peaks associated with impurity phases were present. The morphology analysis showed spherical grains with a random size distribution. The grain sizes increased with the number of BFO layers. The average grain size varied from $43 \mathrm{~nm}$ to $68 \mathrm{~nm}$. The best dielectric results were obtained for the film with 6 LFO sublayers and 4 BFO top layers, in which the dielectric constant showed low dispersion. Since the capacitance-voltage curve for the film 6-LFO/4-BFO is symmetrical around null voltage, it can be inferred that this heterostructure has few mobile ions and accumulated charges on the film-substrate interface. In this film, polarization remains almost constant during $10^{12}$ cycles before the onset of degradation, which shows the very high resistance of the films to fatigue. Magnetoelectric coefficient measurements of the films revealed the formation of hysteresis loops, and a maximum value of $12 \mathrm{~V} / \mathrm{cmOe}$ was obtained for the magnetoelectric coefficient in the longitudinal direction; this value is much higher than that previously reported for pure BFO thin films.
\end{abstract}

(c) 2016 Elsevier Ltd and Techna Group S.r.l. All rights reserved.

\section{Introduction}

The development of multiferroic materials is a new horizon to be explored in the search for high performance and multifunctional devices. State-of-the-art devices can be made more versatile and flexible by adapting new technologies for data storage that offer substantial advantages with respect to energy consumption, speed, and data manipulation. Multiferroic materials $[1,2]$ simultaneously possess ferroic and antiferroic properties, and can be both ferroelectric and ferroelastic [3], as well as ferroelectric and ferromagnetic $[4,5]$. The coexistence of these properties in the same material is attractive for data storage applications, since twice the amount of information can be stored in the same volume, thereby increasing the storage density [6]. For binary data storage in MRAMs [7] and FeRAMs [8], ferromagnetism and ferroelectricity are the two most exploited material properties. Special attention has been paid to ferroelectric thin films with

\footnotetext{
* Corresponding author.

E-mail addresses: drleandrosrr@gmail.com (L.S.R. Rocha), alezipo@yahoo.com (A.Z. Simões).
}

perovskite structure due to their multiple applications, such as dielectrics for capacitors, piezoelectric sensors and transducers, pyroelectric detectors, electrostrictive actuators, accelerometers, and FeRAM and DRAM memories [9-13]. In recent years, the need for efficient transmission and storage data systems has grown exponentially in response to the wide demand for increasing storage volume. There is considerable technological interest targeted towards the miniaturization of these devices at accessible cost, but at the same time, having fast data reading capabilities, and low voltage of operation. Ferroelectric materials show spontaneous and remnant polarization, the direction of which can be reversed by applying an electric field in the direction opposite to that used to polarize the material. This relation between the polarization and applied field is non-linear, and is represented by a typical curve, known as ferroelectric hysteresis. When the electric field is removed, two states of polarization are possible: high remnant polarization $(+\mathrm{Pr})$ and low remnant polarization $(-\mathrm{Pr})$. This makes it possible to create a binary device in the form of a ferroelectric capacitor (metal-ferroelectric-metal) that can be switched electrically. Either of the two states $(+\operatorname{Pr}$ or $-\operatorname{Pr})$ can be designated as 1 or 0 in the computer memory. The polarization retention phenomenon depends on factors such as film thickness, 
nature of the electrodes, grain morphology, annealing temperature, interfacial effects, and stress caused by the substrate. There is, for instance, a lower limit to the film thickness, below which polarization cannot be observed [14].

Unfortunately, materials that are both ferromagnetic and ferroelectric are rare, and the search for a material with large polarization and magnetization at room temperature is still underway. To address this need, the synthesis of multiferroic thin films has been investigated. In multiferroic materials, ferromagnetic and ferroelectric properties coexist at room temperature and also, an intrinsic coupling of ferromagnetism and ferroelectricity is often observed [15,16], which is termed as the magnetoelectric effect. Multiferroic materials are electrically polarized when a magnetic field $\mathrm{H}$ is applied, or, conversely, when an electrical field is applied, it generates a magnetic field in the material [17]. The term multiferroic (or ferroelectromagnetic) is used to describe materials that show two or more primal ferroic effects (ferroelectricity, ferromagnetism or ferroelasticity) occurring in the same phase. The possibility of an ordered coupling of these parameters (polarization, magnetization and spontaneous deformation) makes possible the all-in-one integration of these systems, providing new degrees of freedom to future electronic devices $[18,19]$. Among the well-known multiferroic materials, bismuth ferrites $\left(\mathrm{BiFeO}_{3}\right.$ or BFO) have been studied in-depth. Synthesized for the first time in 1950 by Kiselev, BFO is antiferromagnetic and has a Néel temperature around $630 \mathrm{~K}$. Although promising, BFO has a large leakage current. Over the past few decades, many different methods have been employed to reduce the leakage current, but ideal behavior has not yet been realized for this material. The most promising works show that a hybrid structure based on combining BFO with another ferroelectric material is an interesting alternative to reduce its leakage current, improving its electrical properties, albeit with a lower polarization than the pure BFO thin film [20-23].

Lanthanum ferrites $\left(\mathrm{LaFeO}_{3}\right.$ or LFO) are oxides that crystallize in an orthorhombic distorted perovskite structure with antiferromagnetic properties [24]. These characteristics, in combination with a high Néel temperature, make these materials promising for applications in data storage industry, as components in magnetic/magnetic and magnetic/electric structures [25-28]. Thus, LFO is a strong candidate material to improve the coupling between the magnetization and polarization states in bismuth ferrite. One obstacle to using multiferroic materials at room temperature is the large difference between the ferromagnetic and ferroelectric phase transition temperatures. Other problems that need to be addressed during the production of multiferroics for application in multiple state memories are polarization loss due to fatigue, imprint, and high leakage current and coercive field. Besides, the materials should also show magnetoelectric coupling at room temperature; this coupling can be monitored by applying a magnetic field while simultaneously measuring the saturation polarization and dielectric permittivity [29]. The parameters that are of the greatest interest to multiple state memories are, the values for remnant polarization, which has to be high, and that for coercive field, which must be as low as possible. Other important parameters include dielectric permittivity and leakage current. High dielectric permittivity is desirable, since it ensures a better performance of the capacitor. In parallel, leakage current must be small to avoid data loss, which would lead to a periodic recharge of the capacitor [29].

With the aim to address problems of fatigue, retention, imprint and high current density in multiferroic capacitors, as detailed in the previous paragraph, new strategies have been developed for the growth of heterostructured thin films. The main idea is that, the coupling between the different interfaces, leads to new effects that can be used to create nondestructive data storage in

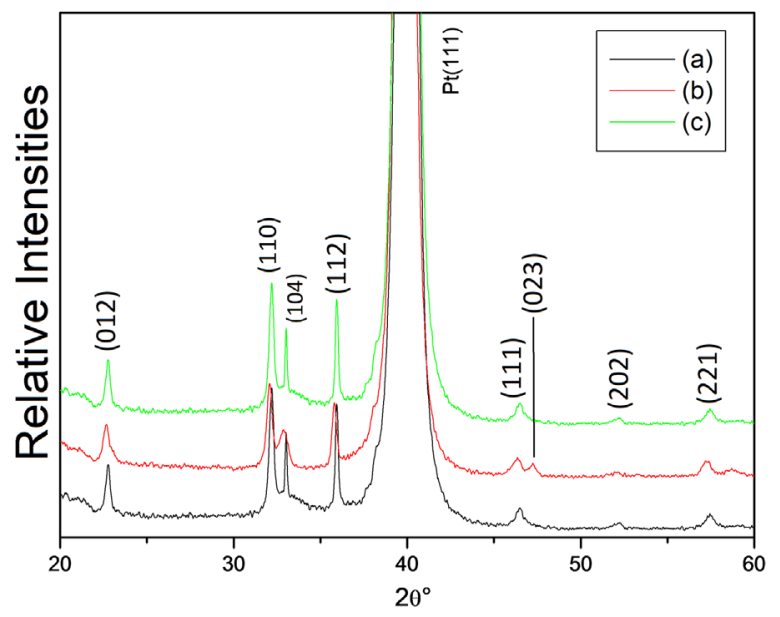

Fig. 1. XRD pattern for the heterostructured thin films deposited above $\mathrm{Si} / \mathrm{Ti} / \mathrm{Pt}$ substrate and annealed at $500{ }^{\circ} \mathrm{C}$ for $2 \mathrm{~h}$. (a) 6LFO/2BFO (b) $6 \mathrm{LFO} / 4 \mathrm{BFO}$ (c) $6 \mathrm{LFO} /$ $6 \mathrm{BFO}$.

memories. This type of interaction has potential applications in the fabrication of multiple state memory elements, in which information can be stored in the polarization state, or in the spontaneously magnetized state, thus leading to revolutionary technology for increased data storage at a high operation speed.

\section{Experimental procedure}

A soft chemical method [24] was used to obtain LFO/BFO thin films. The (100) $\mathrm{Pt} / \mathrm{Ti} / \mathrm{SiO}_{2} / \mathrm{Si}$ substrates were cleaned in an ultrasonic bath after which they were heated to $500{ }^{\circ} \mathrm{C}$ for $1 \mathrm{~h}$ to activate the surface. Thin films were deposited by the spin coating process in which the polymeric precursor resin is dropped onto the substrate and spun at a pre-determined speed for a given time. A commercial spin coater ( $\mathrm{KW}-4 \mathrm{~B}$, Chemat Technology) is used to spin coat the thin films; the operating conditions were set to be $5000 \mathrm{rpm}$ for $30 \mathrm{~s}$. Heat treatment under static air in a conventional oven was carried out to crystallize and densify the films. Each layer was pre-fired at $300^{\circ} \mathrm{C}$ for $1 \mathrm{~h}$, at a heating rate of $3^{\circ} \mathrm{C} /$ min. After pre-firing, the heating rate was increased to $5^{\circ} \mathrm{C} / \mathrm{min}$, reaching $500{ }^{\circ} \mathrm{C}$, maintained for $2 \mathrm{~h}$. The number of LFO sublayers was kept constant while depositing two, four and six layers of BFO resulting in three heterostructures with different thicknesses. The crystallographic phase of the thin films was determined by x-ray diffraction analysis. X-ray diffraction patterns were recorded using a rotating anode tube diffractometer (Rigaku Rotaflex Rint model $2000)$, using $\mathrm{Cu}-\mathrm{K} \alpha$ radiation $(\lambda=1,5406 \AA)$ in the $2 \theta$ range from $20^{\circ}$ to $60^{\circ}$ with steps of $0.3^{\circ} / \mathrm{min}$. Morphological and microstructural characterization of the thin films was carried out using scanning electron microscopy (SEM). Micrographs of the thin films were taken by a field emission gun scanning electron microscope (FEG-SEM), model SUPRA 35-VP (Carl Zeiss), using an operating electron beam voltage of $6 \mathrm{kV}$. Surface morphology and mean grain sizes of the thin films were determined from atomic force microscopy (AFM), in tapping mode (Nanoscope IIIa - Bruker).

For the measurement of electrical properties of the thin films, gold electrodes of $2 \times 10^{-4} \mathrm{~cm}^{2}$ area were sputtered on the surface through a shadow mask with $171 \mu \mathrm{m}$ diameter holes. Dielectric properties, dissipation and resistance were measured as a function of the frequency, using a Hewlett Packard $4194 \mathrm{~A}$ impedance/gain phase analyzer in the frequency range of $100 \mathrm{~Hz}$ to $1 \mathrm{MHz}$, applying a small AC signal of $10 \mathrm{mV}$. Capacitance-Voltage curves were measured by applying a small AC signal of $10 \mathrm{mV}$ and a frequency of $100 \mathrm{kHz}$. The AC signal was measured on the sample 

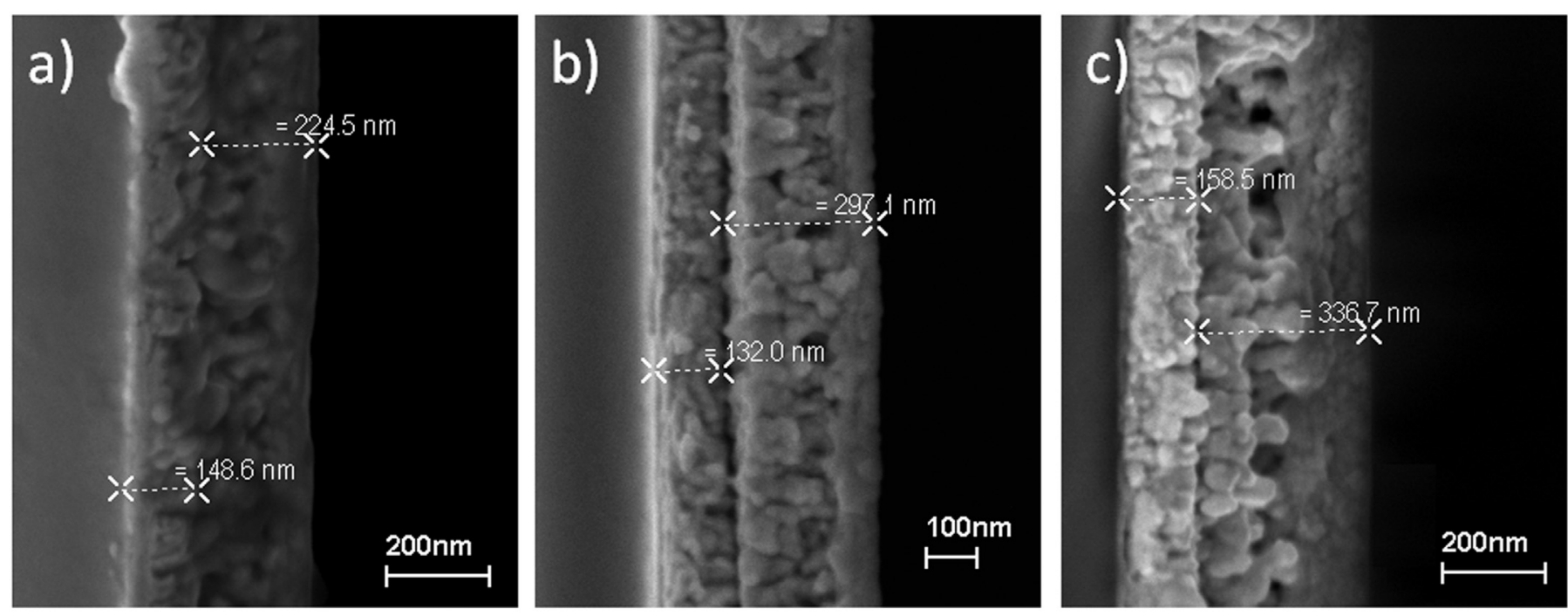

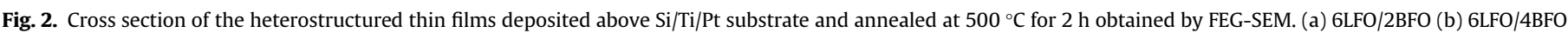
(c) $6 \mathrm{LFO} / 6 \mathrm{BFO}$.

while varying DC signal bias from positive to negative. J-V measurements were recorded on Radiant Technology tester in the current-voltage mode, by changing the voltage from 0 to $+10 \mathrm{~V}$, from +10 to $-10 \mathrm{~V}$ and back to $0 \mathrm{~V}$. Hysteresis loops were measured with the help of a Radiant Technology RT 6000 A equipment composed of a pulse generator and two probes connected to the electrodes, and a computer to process the data.

Magnetoelectric coefficient measurements on the thin films were made using a dynamic lock-in technique. An AC magnetic field up to 10 Oe with a frequency of $7 \mathrm{kHz}$ was superimposed onto the DC field. The AC field was produced by a Helmholtz-type coil (180 turns and $50 \mathrm{~mm}$ diameter), driven by an AC current generated by a function generator (Philips PM5192). The amplitude of the AC field was calculated from the driving current measured by a multimeter (Keithley 196 System DMM). Films were placed in the magnetic field with the surface perpendicular or parallel to the field direction, for longitudinal and transverse measurements, respectively. The DC magnetic bias field was produced by an electromagnet (Cenco Instruments J type). The time-varying DC field was achieved by a programmable DC power supply (Phillips PM2810 $60 \mathrm{~V} / 5 \mathrm{~A} / 60 \mathrm{~W}$ ) and a Hall probe was employed to measure the DC magnetic field.

\section{Results and discussion}

Fig. 1 shows the XRD patterns of the heterostructured thin films produced with two, four and six layers of BFO. The films crystallize in a perovskite structure with no preferential orientation, characteristic of polycrystalline materials. The XRD spectrum can be separated into two sets of well-defined peaks, one belonging to perovskite BFO and the other to LFO. Apart from the peaks related to the substrate ( $\mathrm{Si}$ and $\mathrm{Pt}$ ), no peaks from other phases, such as $\mathrm{Bi}_{2} \mathrm{Fe}_{4} \mathrm{O}_{9}$, were found. Therefore, it is inferred that pure thin films of LFO/BFO were successfully obtained by the soft chemical method. The absence of secondary or impurity phases in the films indicates that little or no chemical reaction/diffusion occurred between the layers of the different materials in the heterostructure. As can be seen from the XRD patterns, it's evident that increasing the BFO layer thickness results in films with higher intensities for the (104) and (112) crystalline planes, and with a low full width at half maximum (FWHM), due to the larger quantity of the crystalline material on the substrate surface.
FEG-SEM images in Fig. 2 show the cross sectional analysis of the LFO/BFO heterostructures. The distinct boundary observed separating the two layers indicates that the BFO thin films were deposited uniquely on the LFO sublayer surface, and no significant diffusion took place between the two distinct phases, which is consistent with the XRD analysis. Also, it can be observed that the film thickness increased with the number of BFO layers. This is due to the large volume of the organic citrate solution retained on the surface for posterior crystallization. The thickness of the Pt layer is around $150 \mathrm{~nm}$, while the thin films vary in thickness from $224.5 \mathrm{~nm}$ for the film with two BFO layers, to $336.7 \mathrm{~nm}$ for the film with six BFO layers.

AFM images showing the surface morphology of the heterostructured thin films, with the corresponding grain sizes are illustrated in Fig. 3. Grains were spherical with a random size distribution. The grain size increased with the number of BFO layers. The surface morphology shows no cracks, and has a low porosity; the average grain size varied from $43 \mathrm{~nm}$ to $68 \mathrm{~nm}$ depending on the number of BFO layers deposited. The heterostructured films exhibited a very homogenous morphology, with no interaction between the sublayers, as previously observed in the SEM images. This observation allows us to conclude that the films are formed by a process of nucleation and growth that results in a homogenous and dense morphology, which in turn, has an effect on the multiferroic properties. In addition, it is clearly seen that increasing the number of BFO layers leads to larger grains and a rougher surface, as shown in Fig. 3-c.

Surface morphologies of heterostructures with LFO as a sublayer are illustrated in Fig. 4. The images show that grain size and morphology are uniform and homogenously distributed. Grain size analysis is essential, since most thin film properties, namely, leakage current, polarization retention and coercive field, difficulties in switching polarization, domain arrangements, and imprint, among others, are strongly influenced by grain size. It is noted that increasing the thickness of BFO layers promoted the densification of the thin films, due to a reduction in intergranular pores. This leads to a thin film with larger grain size with a homogenous size distribution. The plate-type growth typical of BFO layered structure is not observed here; this is due to the presence of a sublayer of LFO between the Pt substrate and the top BFO layer

Fig. 5 shows the dielectric behavior of the heterostructured LFO/BFO thin films as a function of the applied frequency. 
a)
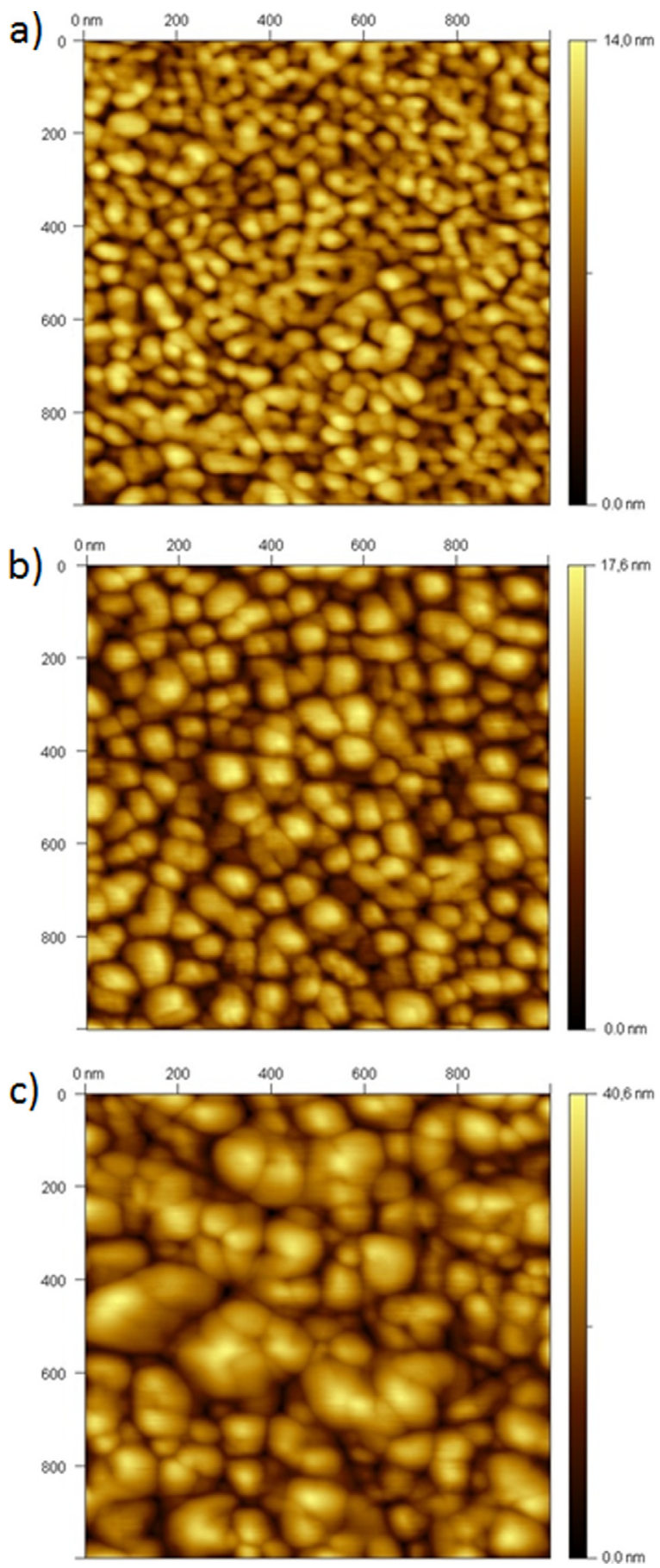

Fig. 3. Micrographies for the heterostructured thin films deposited above $\mathrm{Si} / \mathrm{Ti} / \mathrm{Pt}$ substrate and annealed at $500{ }^{\circ} \mathrm{C}$ for $2 \mathrm{~h}$ obtained by AFM. (a) 6LFO/2BFO (b) 6LFO/ $4 \mathrm{BFO}$ (c) $6 \mathrm{LFO} / 6 \mathrm{BFO}$

Dielectric measurements were carried out at room temperature, in the frequency range of $10 \mathrm{kHz}$ to $1 \mathrm{MHz}$. Fig. 5-a shows the decrease of the dielectric constant with increasing frequency, which could be due to interfacial defects, spatial charge polarization or interface polarization. For the films represented in Fig. 5-b and -c, the dielectric constant does not significantly change with frequency. The sample shown in Fig. 5-b has the highest dielectric constant, which may be due to the reduced number of oxygen vacancies, or may even be due to additional stress introduced between the LFO sublayer and the top BFO layer resulting from both the different lattice parameter of the two materials, and the thermal expansion coefficient of the LFO/BFO interface. The larger the dispersion of the dielectric constant with frequency, the greater is the number of interfacial defects, the presence of which has the following negative effects on the magnetic hysteresis loops: unsaturation, large polarization gaps, absence of reversibility of the magnetic domains, and a lower ferromagnetic coupling factor. In the films shown in 5-b and 5-c, the dielectric constant shows a very low dispersion, while at the same time having a good adherence to the substrate, which implies that the interactions at the heterostructure interface did not generate defects. The addition of LFO sublayer on the Pt substrate reduces the leakage current as compared to a pure BFO film on the same substrate [30]. This lower leakage current can be attributed to a decrease in the number of electrons injected into the cathode, in a way that they move very fast through the thin film. A low electric field was applied to the LFO film in order to overcome the repulsive forces; since these forces are higher due to an increase in the quantity of loaded charges retained. This study demonstrates that the morphology of the ferroelectric films plays an important role in determining the electrical conductivity. Since the conductivity is strongly influenced by the characteristics of the filmelectrode interface due to dielectric phenomena such as the creation of Schottky-type barriers, the low leakage current observed can probably be attributed to differences in grain size and density, and also to lower stress on the film surface owing to differences in the nature of the interface between the ferroelectric material and the electrode. This effect, related to the current density, contributes to the increase in the permittivity of the 6-LFO/4-BFO film (Fig. 5-b).

The capacitance-voltage curves are shown in Fig. 6. All the films show a strongly non-linear dependence on voltage, indicating that the ferroelectric properties originate from domain reversion. Furthermore, the observation that the curve for the 6-LFO/4-BFO film is symmetric around null voltage indicates that this heterostructure has few mobile ions and accumulated charges on the film-substrate interface, even though the presence of crystallographic defects makes it difficult to reverse the domains, leading to polarization saturation. The $C-V$ curves for the films with two and six BFO layers indicate asymmetry in the maximum capacitance values, observed by reversion mechanisms of ferroelectric domains. This suggests the presence of retained charges at the film-electrode interface. Further studies on thin films deposited on oxide electrodes are being carried out, where the leakage current is reduced by avoiding the formation of spatial charges at the filmelectrode interface. It is noteworthy that for some hysteresis loops, saturation occurs at lower voltage values when compared to those determined from $C-V$ curves. This is explained by the difference in the frequencies used to measure $C-V$ curves and hysteresis loops. Polarization and capacitance depend on frequency; thus, the kinetics for ferroelectric domain reversion can be distinctly different, which has a direct influence on the shape of the curves.

Hysteresis loops for the LFO/BFO heterostructures are represented in Fig. 7. At an applied voltage of $10 \mathrm{~V}$, the films show remnant polarization values of $10.97,20.35$ and $13.79 \mu \mathrm{C} / \mathrm{cm}^{2}$, as shown in Fig. 8-a, b and c, respectively. These values are not intrinsic, but are probably induced by the high leakage current in these structures, as seen by the low values for polarization saturation in the $P-V$ curves. Also, a weak hysteresis loop, common in conductive ferroelectrics, is observed. The hysteresis loops at room temperature for the LFO/BFO thin films with four and six BFO layers indicate polarization saturation, and a remnant polarization of $20 \mu \mathrm{C} / \mathrm{cm}^{2}$ was measured after applying a voltage of $10 \mathrm{~V}$. No signal due to leakage current was detected at $60 \mathrm{~Hz}$. A lowering of the coercive field is expected when the thickness of the top BFO layer is increased. The films with more BFO layers had longer annealing times, resulting in higher defect concentrations. The observed remnant polarization of $13.79 \mu \mathrm{C} / \mathrm{cm}^{2}$ at $10 \mathrm{~V}$ for the 

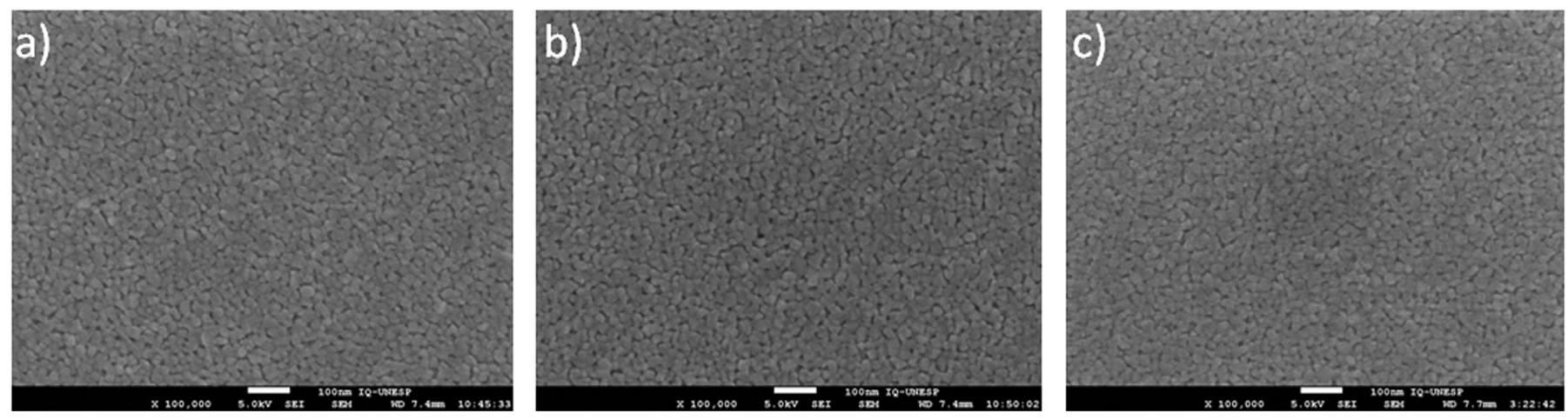

Fig. 4. Micrographies for the heterostructured thin films deposited above Si/Ti/Pt substrate and annealed at $500{ }^{\circ} \mathrm{C}$ for $2 \mathrm{~h}$ obtained by SEM. (a) $6 \mathrm{LFO} / 2 \mathrm{BFO}$ (b) $6 \mathrm{LFO} / 4 \mathrm{BFO}$ (c) 6LFO/6BFO.
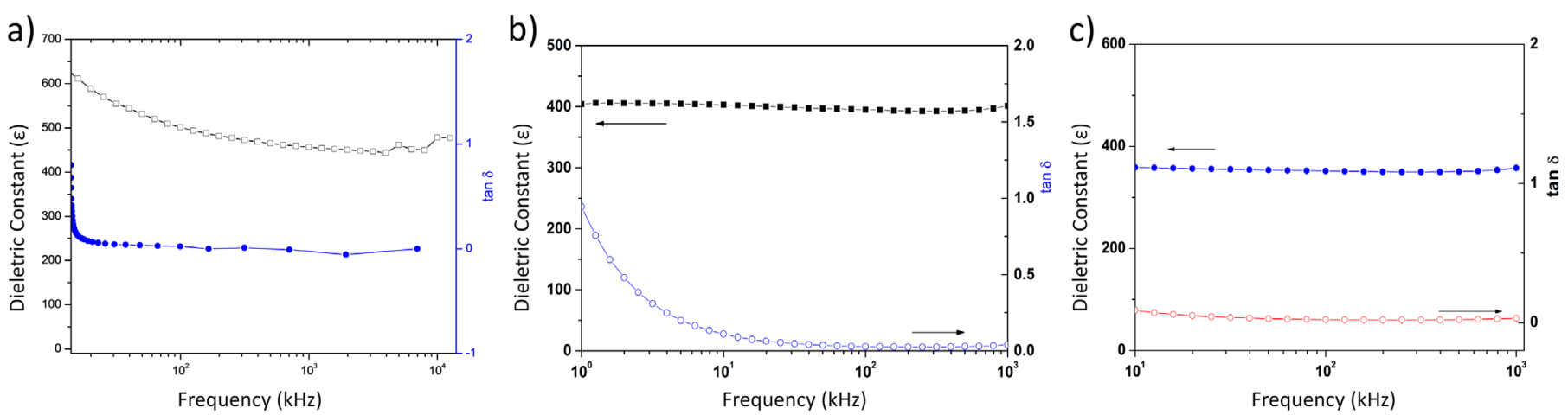

Fig. 5. Dielectric permittivity for the heterostructured thin films deposited above $\mathrm{Si} / \mathrm{Ti} / \mathrm{Pt}$ substrate and annealed at $500{ }^{\circ} \mathrm{C}$ for $2 \mathrm{~h}$. (a) $6 \mathrm{LFO} / 2 \mathrm{BFO}$ (b) $6 \mathrm{LFO} / 4 \mathrm{BFO}$ (c) $6 \mathrm{LFO} /$ $6 \mathrm{BFO}$.
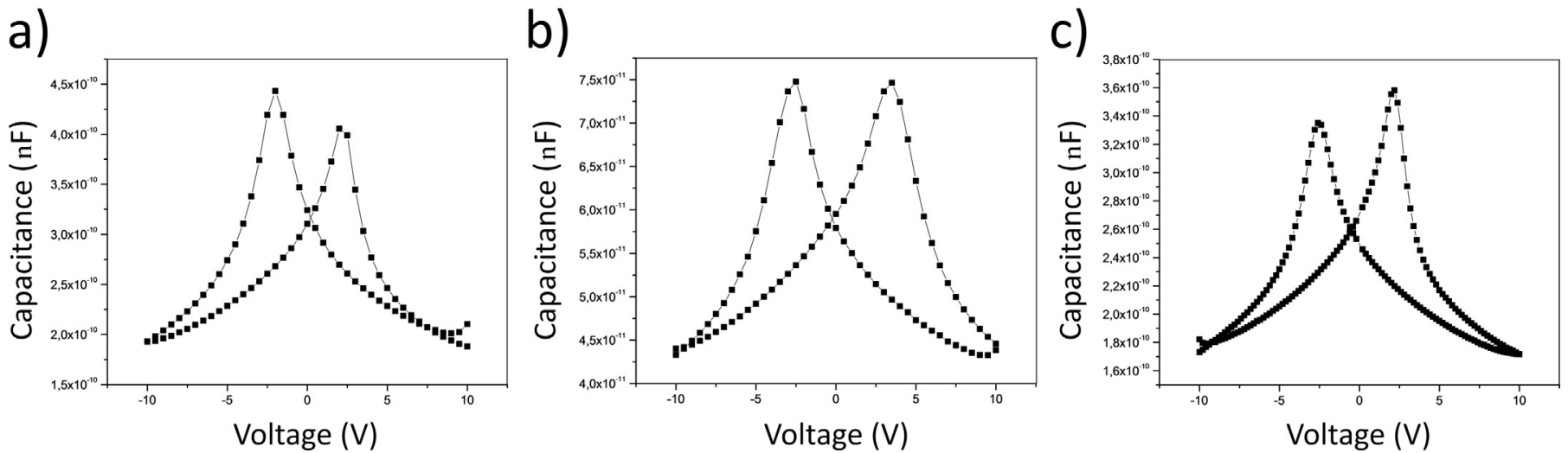

Fig. 6. Capacitance-Voltage curves for the heterostructured thin films deposited above Si/Ti/Pt substrate and annealed at $500{ }^{\circ} \mathrm{C}$ for $2 \mathrm{~h}$. (a) $6 \mathrm{LFO} / 2 \mathrm{BFO}$ (b) $6 \mathrm{LFO} / 4 \mathrm{BFO}$ (c) $6 \mathrm{LFO} / 6 \mathrm{BFO}$
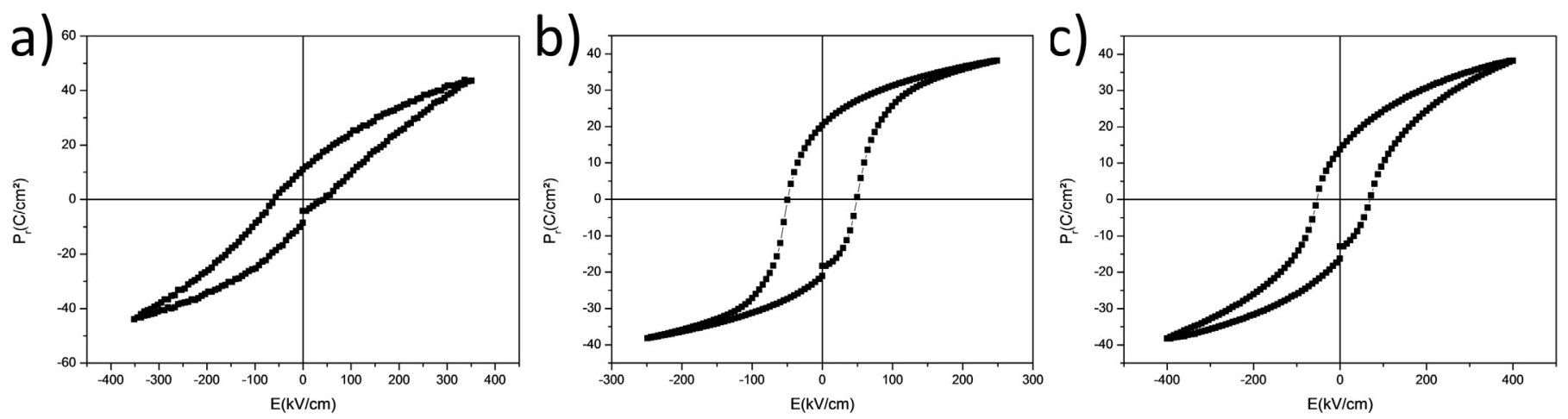

Fig. 7. Ferroelectric hysteresis for the heterostructured thin films deposited above Si/Ti/Pt substrate and annealed at $500{ }^{\circ} \mathrm{C}$ for $2 \mathrm{~h}$. (a) $6 \mathrm{LFO} / 2 \mathrm{BFO}$ (b) $6 \mathrm{LFO} / 4 \mathrm{BFO}$ (c) $6 \mathrm{LFO} /$ $6 \mathrm{BFO}$. 


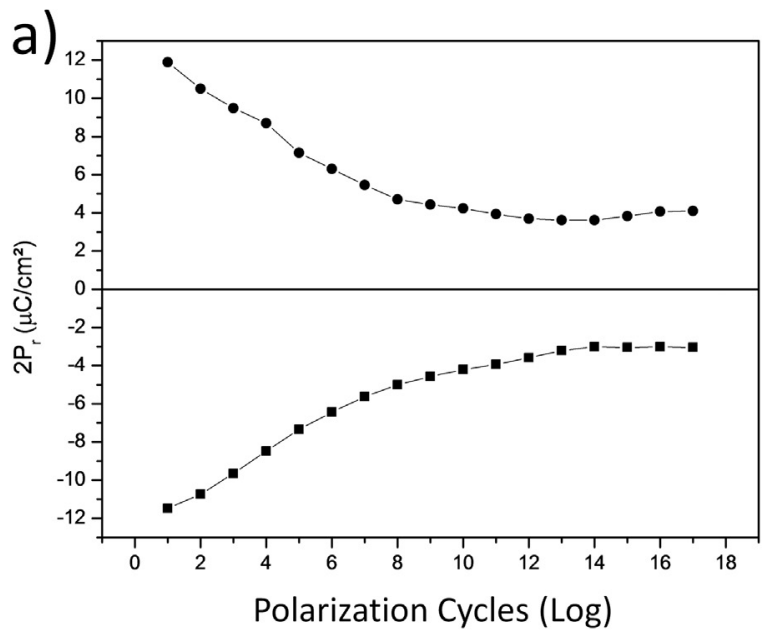

b)
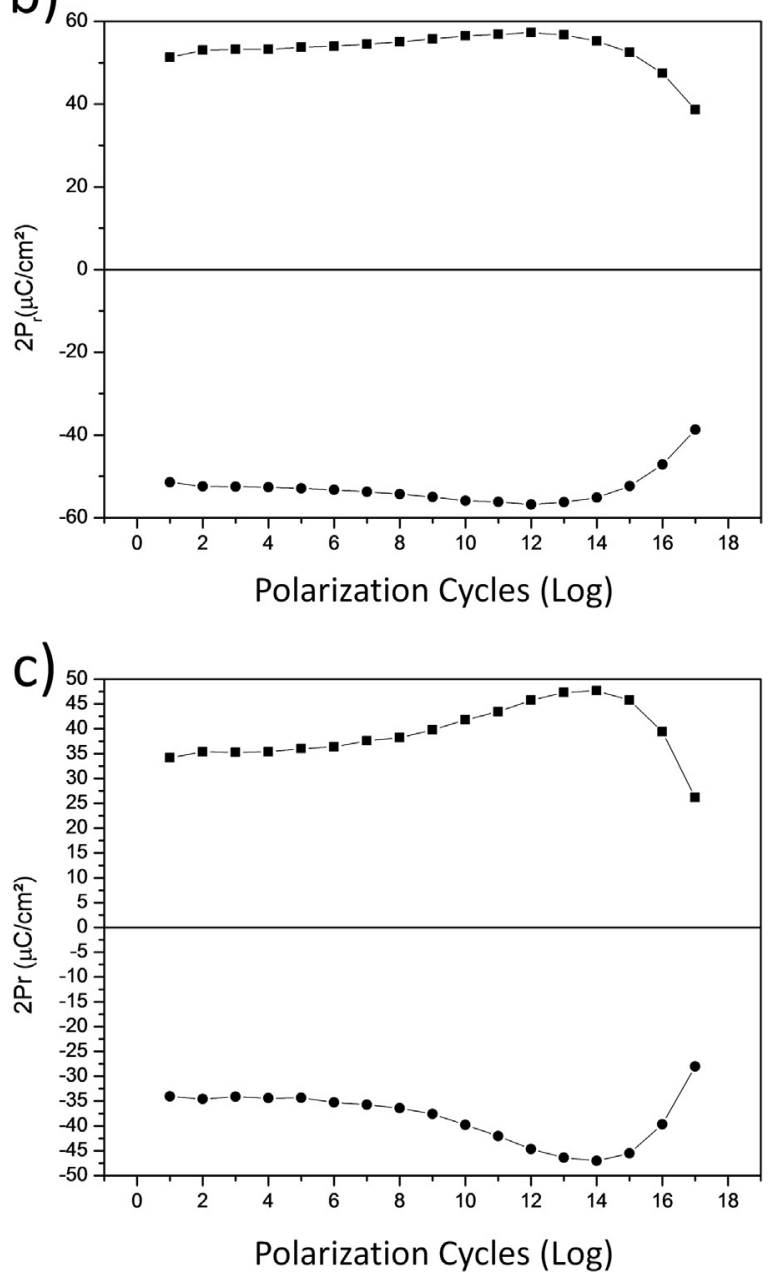

Fig. 8. Fatigue resistance for the heterostructured thin films deposited above $\mathrm{Si} / \mathrm{Ti} /$ Pt substrate and annealed at $500{ }^{\circ} \mathrm{C}$ for 2 h. (a) 6LFO/2BFO (b) $6 \mathrm{LFO} / 4 \mathrm{BFO}$ (c) $6 \mathrm{LFO}$ $6 \mathrm{BFO}$.

heterostructure with six top layers reveals that, after a certain thickness, defects tend to reach equilibrium in the crystalline lattice, as a result of which, the domains occupy energetically favorable positions without modifying the nature of the $P-E$ curve. Fig. 7-a shows a displacement along the coercive field axis in the positive direction. This phenomenon is known as imprint. Voltage fluctuations can damage the capacitor due to the apparent polarization loss in one of the reminiscent states as a result of which, a)

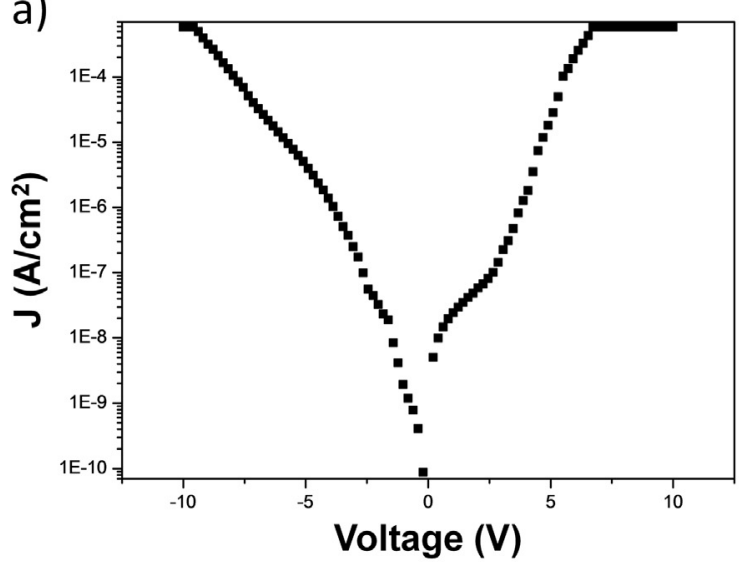

b)

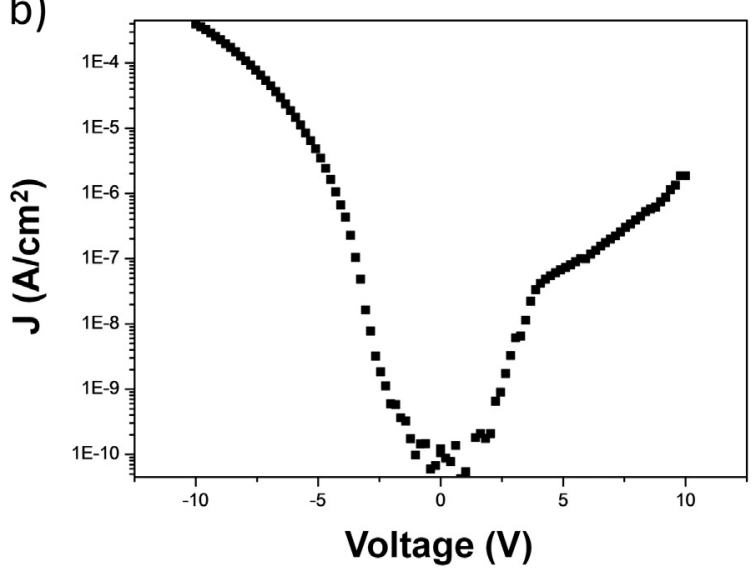

c)

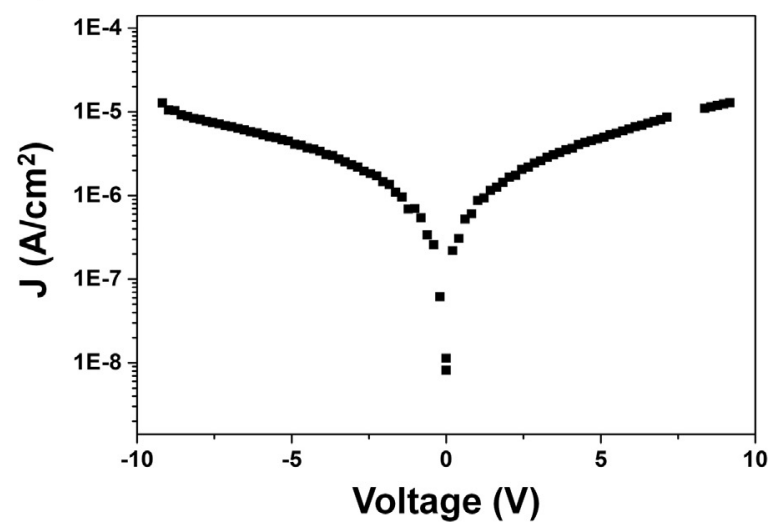

Fig. 9. Current density curves for the heterostructured thin films deposited above $\mathrm{Si} / \mathrm{Ti} / \mathrm{Pt}$ substrate and annealed at $500{ }^{\circ} \mathrm{C}$ for $2 \mathrm{~h}$. (a) $6 \mathrm{LFO} / 2 \mathrm{BFO}$ (b) $6 \mathrm{LFO} / 4 \mathrm{BFO}$ (c) 6LFO/6BFO

the coercive field can increase along one direction. These effects may cause capacitor memory failure, and are coherent with the $C$ $V$ and $I-V$ measurement data. The origin of the electrical conductivity, which is critical to understand the conductive behavior may be traced to oxygen vacancies, created to maintain electrical neutrality. In $\mathrm{BiFeO}_{3}$ samples, oxygen vacancies increasingly ionize to compensate the gaps introduced by the addition of the sublayer. Oxygen vacancies are mobile positive charges that can move through the sample under high electric fields to maintain thermodynamic equilibrium. This results in a charge imbalance between the donor oxygen and the receiver lanthanum ions enabling n-type charge transport on the surface, while the region near the electrode becomes p-type. Applying an electric field opposite to 
a)
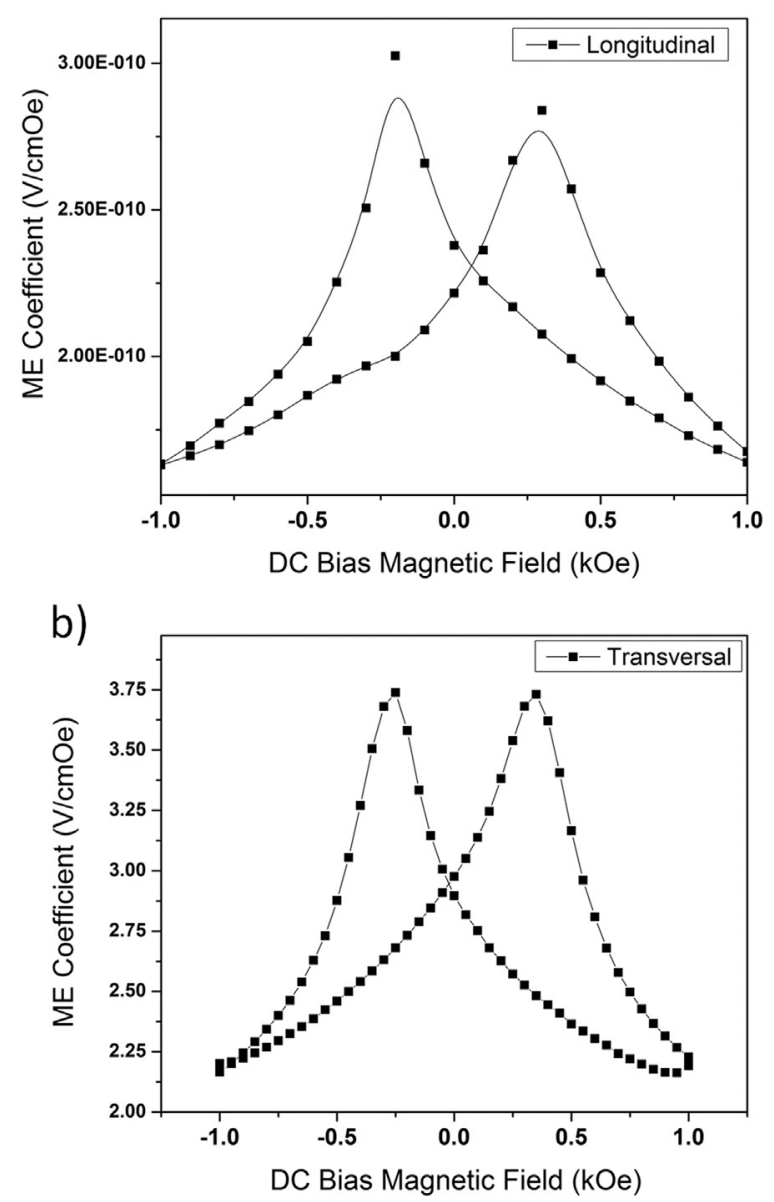

Fig. 10. Magnetoelectric coupling curves for the heterostructured thin film with $6 \mathrm{LFO} / 4 \mathrm{BFO}$ configuration deposited above $\mathrm{Si} / \mathrm{Ti} / \mathrm{Pt}$ substrate and annealed at $500{ }^{\circ} \mathrm{C}$ for $2 \mathrm{~h}$. (a) Longitudinal (b) Transversal.

the oxygen vacancies flux, they migrate to gaps, becoming an electronic insulator.

The variation of fatigue with the number of polarization cycles is represented in Fig. 8. The heterostructured LFO/BFO thin films were analyzed under the application of bipolar pulses of $8.6 \mu$ s of $10 \mathrm{mV}$ amplitude. Remnant polarization is calculated by the difference between the reverse and non-reverse polarizations. In ferroelectric thin films, the common causes of fatigue are the formation of a top layer, difficulties in switching domains due to the presence of accumulated defects in the region, difficulties in polarization due to volume defects, suppression of the nucleation of the oppositely oriented domain at the surface, and damage to the film-electrode interface. For the film shown in Fig. 8-a, an excellent fatigue resistance was achieved, around $10^{15}$ polarization cycles. However, this sample not only has a small polarization, but also shows a decrease in the remnant polarization by more than $50 \%$ at $10^{8}$ cycles. In Fig. 8 -b, it is seen that the polarization remains almost constant during $10^{12}$ cycles before the onset of degradation. For the sample shown in Fig. 8-c, a different behavior is observed where it is seen that the remnant polarization strongly increases around $10^{8}$ cycles; this effect can be explained to be due to the suppression of the nucleation of oppositely charged domains at the surface. This increase in the remnant polarization leads to a higher local conductivity of the films in the region containing these nucleation sites. The fall and the consequent degradation of the film around $10^{14}$ cycles is probably due to local increase in current, which eventually damages the film-electrode interface, diminishing the number of cycles of ferroelectric domain reversion.

$J-V$ measurements were recorded using the Radiant Technology tester in the current-voltage mode, by increasing the voltage from 0 to $+10 \mathrm{~V}$, switching from +10 to $-10 \mathrm{~V}$ and decreasing the voltage back to $0 \mathrm{~V}$ (Fig. 9). The curves were traced with steps of $0.1 \mathrm{~V}$ with a time of $1.0 \mathrm{~s}$ at each voltage. Initially, the current density rises linearly with electric field, indicating ohmic conduction. For higher voltages, the current density increases exponentially, implying that at least a part of the conductivity results from a Schottky or Poole-Frenkel type emission. It is also observed that the current density at $1 \mathrm{~V}$ decreases as the BFO top layer thickness is increased. The factors that contribute to this improvement are described below. The average grain size increases with BFO thickness. Since the conductivity is strongly affected by the characteristics of the metal-semiconductor contact, the film morphology strongly influences the changes in current density in ferroelectric capacitors. Another explanation for the decrease in current density is the reduction of micro-cracks caused by residual stress. Since lattice stress and phase transition vary along the bismuth layer thickness, the unit cell volume decreases during cooling and the residual stress is increased. The slight drop in the current density values can also be attributed to the difference in the sizes and shapes of the grains, their density and the surface structure. Thus, the characteristics of the film-electrode interface, and the surface morphology of the BFO films, predominantly determine the current density in metal-ferroelectric-metal like capacitors.

The plot of the magnetoelectric coefficient as a function of the polarization magnetic field in longitudinal and transversal directions reveals hysteretic behavior for the 6-LFO/4-BFO heterostructured film, as can be seen in Fig. 10a and b. The maximum observed magneto-electric coefficient of $12 \mathrm{~V} / \mathrm{cmOe}$ in the longitudinal direction, is much higher than the values previously reported for pure BFO thin films ( $3 \mathrm{~V} / \mathrm{cmOe}$ ) in the same direction as a consequence of the antiferromagnetic axis of the artificial heterostructure, which rotates through the crystal with an incommensurate speed for a wavelength period of approximately 620 Å. Early reports showed that the spiral spin structure leads to a cancellation of any macroscopic magnetization and would hinder the observation of the linear magnetoelectric effect [31]. A significant magnetization, around $0.5 \mu \mathrm{B}$ per unit cell, and a strong magnetoelectric coupling, can be expected in films under stress, suggesting that the spiral spin structure can be suppressed.

\section{Conclusions}

A composite-like multiferroic heterostructure composed of lanthanum ferrite (LFO) and bismuth ferrite (BFO) was successfully synthesized by a soft chemical method. The crystalline thin films have the perovskite structure. XRD patterns indicate two distinct phases characteristic of LFO and BFO. Except for peaks related to the substrate, no additional secondary phases are observed, demonstrating the purity of the films. Cross sectional analysis of the heterostructure reveals that the films are deposited uniquely on the substrate and on the LFO sublayer. Thicknesses of $224.5 \mathrm{~nm}$ and $336.7 \mathrm{~nm}$ were measured for the 6 -LFO/2-BFO and 6-LFO/6BFO thin films, respectively, (substrate thickness of around $150 \mathrm{~nm}$, is not taken into consideration). Surface morphology showed homogenously distributed grains, and the average grain size increased with the number of BFO layers. The surface is free from cracks and has low porosity. The dielectric constant gradually decreases with frequency for the 6-LFO/2-BFO film, indicating interfacial defects and polarization of spatial charges, or interface polarization. The film with the composition 6-LFO/6-BFO showed 
the best dielectric behavior, with a high dielectric constant and a low variation with frequency, probably due to oxygen vacancies or stress introduced at the interface by the different lattice parameters of LFO and BFO. The low dispersion of $6-\mathrm{LFO} / 4-\mathrm{BFO}$ and 6-LFO/6-BFO thin films indicate good adherence to the substrate and the absence of interface defects. Fatigue analysis presented excellent outcomes. The best result was for the film with the composition 6-LFO/4-BFO, in which reminiscent polarization remained almost unaltered up to $10^{12}$ cycles after which degradation started. Though the other films also presented good results for fatigue resistance, reminiscent polarization fell by more than $50 \%$ during cycling for the 6-LFO/2-BFO film, and for the 6-LFO/6-BFO film, polarization increased due to the suppression of oppositely oriented domain nucleation at the surface, which resulted in a higher conductivity of the films locally, in the region with nucleation sites. The maximum magnetoelectric coefficient of $12 \mathrm{~V} /$ cmOe in the longitudinal direction is a consequence of the antiferromagnetic axis of the artificial heterostructure, which spins at an incommensurate speed through the crystal.

\section{Acknowledgments}

The authors gratefully acknowledge the financial support of the São Paulo Research Foundation (FAPESP) (Grant CEPID/CDMF-FAPESP: 2013/07296-2) and The National Council for Scientific and Technological Development (CNPq) (Grant: 573636/2008-7) and INCTMN (2008/57872-1). The authors also thank the LMA-IQ for providing the FEG-SEM facilities.

\section{References}

[1] G.A. Smolenskii, I.E. Chupis, Ferroelectromagnetics, Sov. Phys. Uspekhi 25 (1982) 475-493.

[2] W. Eerenstein, F.D. Morrison, J. Dho, M.G. Blamire, J.F. Scott, Comment on epitaxial $\mathrm{BiFeO}_{3}$ multiferroic thin film heterostructures, Science 307 (2005) 1203.

[3] A. Vasudevarao, A. Kumar, L. Tian, J.H. Haeni, Y.L. Li, C.J. Eklund, Q.X. Jia, R. Uecker, P. Reiche, K.M. Rabed, L.Q. Chen, D.G. Schlom, G. Venkatraman, Multiferroic domain dynamics in strained strontium titanate, Phys. Rev. Lett. 97 (2006) 257602.

[4] K. Kato, S. lida, Observation of ferroelectric hysteresis loop of $\mathrm{Fe}_{3} \mathrm{O}_{4}$ at $4.2 \mathrm{~K}$, J. Phys. Soc. Jpn. 51 (1982) 13351336.

[5] Y. Yamasaki, S. Miyasaka, Y. Kaneko, J.P. He, T. Arima, Y. Tokura, Magnetic reversal of the ferroelectric polarization in a multiferroic spinel oxide, Phys. Rev. Lett. 96 (2006) 207204

[6] M. Gajek, M. Bibes, S. Fusil, K. Bouzehouane, J. Fontcuberta, A. Barthélémy, A. Fert, Tunnel junctions with multiferroic barriers, Nat. Mater. 6 (2007) 296-302.

[7] W.J. Gallagher, S.S.P. Parkin, Development of the magnetic tunnel junction MRA Mat IBM: from first junctions to a 16-Mb MRAM demonstrator chip, IBM] Res. Dev. 50 (2006) 5-23.

[8] M. Dawber, K.M. Rabe, J.F. Scott, Physics of thin-film ferroelectric oxides, Rev.
Mod. Phys. 77 (2005) 1083-1130

[9] R. Ramesh, A. Inam, W.K. Chan, B. Wilkens, K. Remschning, D.L. Hart, J. M. Tarascon, Epitaxial cuprate superconductor ferroelectric heterostructures, Science 252 (1991) 944-946.

[10] T. Nagahama, T. Manabe, I. Yamaguchi, T. Kumagai, T. Tsuchiya, S. Mizuta, Epitaxy of (106)-oriented $\mathrm{SrBi}_{2} \mathrm{Ta}_{2} \mathrm{O}_{9}$ and $\mathrm{SrBi}_{2} \mathrm{Nb}_{2} \mathrm{O}_{9}$ thin films, Thin Solid Films 353 (1999) 52-55.

[11] S.Y. Wu, W.J. Takei, M.H. Francombe, S.E. Cummins, Domain structure and polarization reversal in films of ferroelectric bismuth titanate, Ferroelectrics 3 (1972) 217-224.

12] S. Choopun, T. Matsumoto, T. Kawai, Low-temperature growth of $\mathrm{Bi}_{4} \mathrm{Ti}_{3} \mathrm{O}_{12}$ epitaxial films on $\mathrm{SrTiO}_{3}(001)$ and $\mathrm{Bi}_{2} \mathrm{Sr}_{2} \mathrm{CaCu}_{2} \mathrm{O}_{8}(001)$ single crystals by lase molecular beam epitaxy, Appl. Phys. Lett. 67 (1995) 1072-1074.

[13] H. Irie, M. Miyayama, T. Kudo, Structure dependesse of ferroelectric properties of bismuth layer-structured ferroelectric single crystals, J. Appl. Phys. 90 (2001) 4089-4094.

[14] C.G. Pinto, M.C.L. Silva, Seminário de Eletrônica e Instrumentação: Memória Ferroelétrica, Escola de Engenharia de Lorena, USP, Lorena, 2009.

[15] A.D. Rae, J.G. Thompson, R.L. Withers, A.C. Willis, Structure refinement of commensurately modulated bismuth titanate, $\mathrm{Bi}_{4} \mathrm{Ti}_{3} \mathrm{O}_{12}$, Acta Cryst. B 46 (1990) 474-487.

[16] S.W. Cheong, M. Mostovoy, Multiferroics: a magnetic twist for ferroelectricity, Nat. Mater. 6 (2007) 13-20.

[17] J.G. Wan, J.M. Liu, H.L.W. Chand, C.L. Choy, H.G. Wang, C.W. Nan, Giant magnetoelectric effect of a hybrid of magnetostrictive and piezoelectric composites, J. Appl. Phys. 93 (2003) 9916-9919.

[18] H. Schmid, Multi-ferroic magnetoelectrics, Ferroelectrics 162 (1994) 317-338

[19] M. Fiebig, T. Lottermoser, D. Frohlich, A.V. Goltsev, R.V. Pisarev, Observation of coupled magnetic and electric domains, Nature 419 (2002) 818-820.

[20] J.G. Wu, G.Q. Kang, H.J. Liu, J. Wang, Ferromagnetic, ferroelectric, and fatigue behavior of (111)-oriented $\mathrm{BiFeO}_{3} /\left(\mathrm{Bi}_{1 / 2} \mathrm{Na}_{1 / 2}\right) \mathrm{TiO}_{3}$ lead-free bilayered thin films, Appl. Phys. Lett. 94 (2009) 172906.

[21] J.G. Wu, G.Q. Kang, J. Wang, Electrical behavior and oxygen vacancies in $\mathrm{BiFeO}_{3} /\left[\left(\mathrm{Bi}_{1 / 2} \mathrm{Na}_{1 / 2}\right)_{99_{4}} \mathrm{Ba}_{0.66}\right] \mathrm{TiO}_{3}$ thin film, Appl. Phys. Lett. 95 (2009) 192901

[22] H. Béa, M. Bibes, S. Fusil, K. Bouzehouane, E. Jacquet, K. Rode, P. Bencok, A. Barthélémy, Investigation on the origin of the magnetic moment of $\mathrm{BiFeO}_{3}$ thin films by advanced x-ray characterizations, Phys. Rev. B 74 (2006) 020101 (R).

[23] D. Xie, Y. Zang, Y. Luo, X. Han, T. Ren, L. Liu, Structural, ferroelectric, dielectric and magnetic properties of $\mathrm{BiFeO}_{3} / \mathrm{Bi}_{3.15} \mathrm{Nd}_{0.85} \mathrm{Ti}_{3} \mathrm{O}_{12}$ multilayer films derived by chemical solution deposition, J. Appl. Phys. 105 (2009) 084109.

[24] D. Kuscer, M. Hrovat, J. Holc, S. Bernik, D. Kolar, Some characteristics of $\mathrm{A}_{12} \mathrm{O}_{3}$ and $\mathrm{CaO}$-modified $\mathrm{LaFeO}_{3}$-based cathode materials for solid oxide fuel cells, J Power Sources 61 (1996) 161-165.

[25] R. Dogra, A.C. Junqueira, R.N. Saxena, A.W. Carbonari, J.M. Filho, M. Moralles, Hyperfine interaction measurements in $\mathrm{LaCrO}_{3}$ and $\mathrm{LaFeO}_{3}$ perovskites using perturbed angular correlation spectroscopy, Phys. Rev. B 63 (2001) 224104.

[26] A. Scholl, J. Stohr, J. Luning, J.W. Seo, J. Fompeyrine, H. Siegwart, J.P. Locquet, F. Nolting, S. Anders, E.E. Fullerton, M.R. Scheinfein, H.A. Padmore, Observation of antiferromagnetic domains in epitaxial thin films, Science 287 (2000) $1014-1016$

[27] J. Nogues, I.K. Schuller, Exchange bias, J. Magn. Magn. Mater. 192 (1999) 203-232.

[28] D.A. Pan, J. Lu, Y. Bai, W.Y. Chu, L.J. Qiao, Shape demagnetization effect on layered magnetoelectric composites, Chin. Sci. Bull. 3 (2008) 2124-2128.

[29] S. Bhattacharjee, E. Bousquet, P. Ghosez, Engineering multiferroism in $\mathrm{CaMnO}_{3}$, Phys. Rev. Lett. 102 (2009) 117602.

[30] E.C. Aguiar, M.A. Ramirez, J.A. Cortes, E. Borsari, A.Z. Simões, Magnetoelectric coupling of $\mathrm{LaFeO}_{3} / \mathrm{BiFeO}_{3}$ heterostructures, Ceram. Int. 41 (2015) $13126-13134$.

[31] Y.F. Popov, A.K. Zvezdin, G.P. Vorbev, A.M. Kadomtseva, V.A. Murashev, D. N. Racov, Linear magnetoelectric effect and phase transitions in bismuth ferrite, $\mathrm{BiFeO}_{3}$, JETP Lett. 57 (1993) 69. 